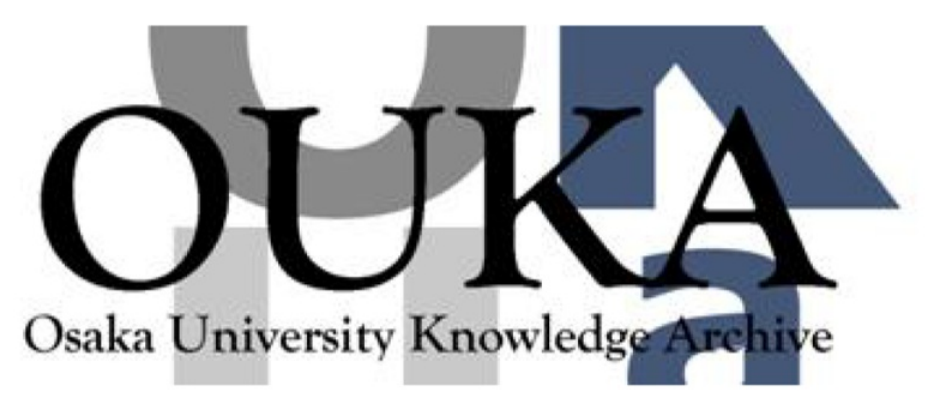

\begin{tabular}{|c|l|}
\hline Title & $\begin{array}{l}\text { Fast growing double tearing modes in a tokamak } \\
\text { plasma }\end{array}$ \\
\hline Author(s) & $\begin{array}{l}\text { Bierwage, Andreas; Benkadda, Sadruddin; } \\
\text { Hamaguchi, Satoshi et al. }\end{array}$ \\
\hline Citation & Physics of Plasmas. 12(8) p. 082504 \\
\hline Issue Date & $2005-08$ \\
\hline oaire:version & VoR \\
\hline URL & $\begin{array}{l}\text { https://hdl. handle. net/11094/78483 } \\
\text { This article may be down loaded for personal use } \\
\text { only. Any other use requi res prior permission } \\
\text { of the author and AIP Publishing. This article } \\
\text { appeared in Phys ics of Plasmas 12, 082504 } \\
\text { (2005) and may be found at } \\
\text { https://doi. org/10.1063/1.1989727. }\end{array}$ \\
\hline rights \\
\hline Note & \begin{tabular}{l}
$\mid$ \\
\hline
\end{tabular} \\
\hline
\end{tabular}

Osaka University Knowledge Archive : OUKA

https://ir. Library. osaka-u. ac. jp/

0saka University 


\section{Fast growing double tearing modes in a tokamak plasma}

Cite as: Phys. Plasmas 12, 082504 (2005); https://doi.org/10.1063/1.1989727

Submitted: 09 March 2005 . Accepted: 09 June 2005. Published Online: 25 July 2005

Andreas Bierwage, Sadruddin Benkadda, Satoshi Hamaguchi, and Masahiro Wakatani

\section{ARTICLES YOU MAY BE INTERESTED IN}

Linear analysis of the double-tearing mode

The Physics of Fluids 23, 1368 (1980); https://doi.org/10.1063/1.863151

Nonlinear evolution of double tearing modes

Physics of Plasmas 7, 4477 (2000); https://doi.org/10.1063/1.1315304

Dynamics of resistive double tearing modes with broad linear spectra

Physics of Plasmas 14, 022107 (2007); https://doi.org/10.1063/1.2446420

\section{AlP Advances Fluids and Plasmas Collection}




\title{
Fast growing double tearing modes in a tokamak plasma
}

\author{
Andreas Bierwage ${ }^{\text {a) }}$ \\ Graduate School of Energy Science, Kyoto University, Gokasho, Uji, Kyoto 611-0011, Japan
}

Sadruddin Benkadda ${ }^{\text {) }}$

Equipe Dynamique des Systèmes Complexes, UMR 6633 CNRS-Université de Provence, 13397 Marseille, France

Satoshi Hamaguchi ${ }^{\mathrm{c})}$

Graduate School of Engineering, Osaka University, 2-1 Yamadaoka, Suita, Osaka 565-0871, Japan

Masahiro Wakatani ${ }^{\text {d) }}$

Graduate School of Energy Science, Kyoto University, Gokasho, Uji, Kyoto 611-0011, Japan

(Received 9 March 2005; accepted 9 June 2005; published online 25 July 2005)

\begin{abstract}
Configurations with nearby multiple resonant surfaces have broad spectra of linearly unstable coupled tearing modes with dominant high poloidal mode numbers $m$. This was recently shown for the case of multiple $q=1$ resonances [Bierwage et al., Phys. Rev. Lett. 9465001 (2005)]. In the present work, similar behavior is found for double tearing modes (DTM) on resonant surfaces with $q \geqslant 1$. A detailed analysis of linear instability characteristics of DTMs with various mode numbers $m$ is performed using numerical simulations. The mode structures and dispersion relations for linearly unstable modes are calculated. Comparisons between low- and higher- $m$ modes are carried out, and the roles of the inter-resonance distance and of the magnetic Reynolds number $S_{\mathrm{Hp}}$ are investigated. High- $m$ modes are found to be destabilized when the distance between the resonant surfaces is small. They dominate over low- $m$ modes in a wide range of $S_{\mathrm{Hp}}$, including regimes relevant for tokamak operation. These results may be readily applied to configurations with more than two resonant surfaces. (C) 2005 American Institute of Physics. [DOI: 10.1063/1.1989727]
\end{abstract}

\section{INTRODUCTION}

Double tearing modes (DTM) are coupled tearing modes on adjacent resonant surfaces which effectively "drive each other." ${ }^{,-3}$ The resulting instability is stronger than a single tearing mode (STM), whereby modes with high poloidal mode numbers $m$ may become dominant. ${ }^{4}$

DTMs are thought to be involved in a variety of dynamical processes in tokamak plasmas. In the current-ramp-up phase of the tokamak operation they were used to explain the anomalously strong current penetration ${ }^{5-7}$ and shortwavelength magnetohydrodynamic (MHD) activity (e.g., Mirnov oscillations). ${ }^{6,8}$ Experimental observations of partial and compound sawtooth crashes (internal disruptions) ${ }^{9-13}$ may be explained through kink-tearing modes on double/ multiple resonant surfaces. ${ }^{14-17}$ Phenomena associated with low-beta disruptions in the presence of double resonant surfaces with $q_{\mathrm{s}}>1$ may also be understood in terms of DTM activity. ${ }^{14,18,19}$ In some way related to DTMs is the coupling between modes on resonant surfaces with different $q_{\mathrm{s}}$, which may explain some salient features of major disruptions. ${ }^{7,20,21}$ A more recent application is related to the formation of internal transport barriers (ITB) in advanced tokamaks. DTMs were suggested to be involved in this process due to the fact that ITBs are often observed in the vicinity of resonant surfaces or near $q_{\text {min. }}{ }^{22-24}$

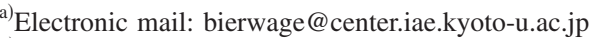

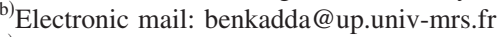

${ }^{c)}$ Electronic mail: hamaguch@ppl.eng.osaka-u.ac.jp

${ }^{\mathrm{d})}$ Deceased.
}

It was found that DTMs with any mode number $m$ become similar to $m=1$ internal kink modes ${ }^{25}$ when the distance between the resonant surfaces is sufficiently small. ${ }^{6}$ This is reflected by the linear growth rate $\gamma_{\text {lin }}$ scaling with the magnetic Reynolds number $S_{\mathrm{Hp}}$ as $\gamma_{\operatorname{lin}} \propto S_{\mathrm{Hp}}^{-1 / 3}$. When the distance between the resonant surfaces is large, the structure of the instability resembles that of individual STMs localized on each resonant surface. In this case, the scaling law is $\gamma_{\text {lin }} \propto S_{\mathrm{Hp}}^{-3 / 5} \cdot{ }^{26}$ Furthermore, it was found that the coupling in a DTM may also destabilize linearly stable tearing modes ${ }^{18}$ and speed up the growth of magnetic islands in the nonlinear regime. ${ }^{1,19,27,28}$ The instability of DTMs may be enhanced by effects like anomalous electron viscosity, ${ }^{6,29}$ finite beta and sheared toroidal flows. ${ }^{30}$ Poloidal shear flows, on the other hand, may have a stabilizing effect, ${ }^{18,31,32}$ raising the possibility of a dynamical interplay between shear flows and DTMs. ${ }^{18,33}$ This idea is supported by recent studies which indicate that MHD activity associated with multiple resonant surfaces may produce a significant amount of sheared poloidal flows. ${ }^{29,30}$

Until now, studies related to DTMs in cylindrical (or toroidal) plasmas were focusing mainly on the role of the modes with the lowest poloidal and toroidal mode numbers, $m$ and $n$. High- $m$ tearing modes were associated only with correspondingly high values of $q_{\mathrm{s}}=m / n .^{1,6,8,34}$ Recently it was demonstrated for the case of $q_{\mathrm{s}}=1$ double and triple tearing modes that modes with high $m$ may dominate over low- $m$ modes in a cylindrical plasma even on low- $q$ resonant surfaces. ${ }^{4}$ There it was found that in configurations where the distance between neighboring resonant surfaces is suffi- 
ciently small, broad spectra of unstable modes exist and that the dominant modes can have $m \sim \mathcal{O}(10)$.

Motivated by the results of Ref. 4, in the present work we investigate the conditions under which broad spectra of multiple tearing modes with dominant higher- $m$ modes may arise. For this purpose numerical simulations based on the reduced resistive magnetohydrodynamic model in cylindrical geometry are used. The dependence of the linear growth rate on parameters such as the inter-resonance distance, the dissipation coefficients as well as the magnetic shear will be addressed.

The main results of the present work are the following. It is shown that broad spectra of unstable DTMs are also found in configurations with $q_{\mathrm{s}}>1$, thus generalizing the results of Ref. 4. The instability of modes with $m>1$ depends strongly on the distance between adjacent resonant surfaces. For the resistivity dependence, a scaling law $\gamma_{\operatorname{lin}} \propto S_{\mathrm{Hp}}^{-\alpha}$ is found with $1 / 3 \leqslant \alpha \leqslant 3 / 5$, which is valid in a range of high values of $S_{\mathrm{Hp}}$. This is in agreement with former studies. ${ }^{2,28}$ For intermediate values of $S_{\mathrm{Hp}}$ no power law is identified and for low $S_{\mathrm{Hp}}$ it is found that $\gamma_{\text {lin }} \propto S_{\mathrm{Hp}}$ independently of $m$. For high $m$ and small $D_{12}$ the range where the relation $\gamma_{\text {lin }} \propto S_{\mathrm{Hp}}^{-\alpha}$ does not hold can be rather wide, and it is especially in this regime where high- $m$ modes are found to be dominant. This regime may include ranges of $S_{\mathrm{Hp}}$ that are relevant to tokamak operation.

The present paper is organized as follows. Section II contains an introduction of the model equations and the numerical method used. Numerical results are presented in Sec. III, followed by a discussion and conclusions in Sec. IV.

\section{MODEL}

\section{A. Reduced MHD equations}

The reduced set of magnetohydrodynamic (RMHD) equations in cylindrical geometry ${ }^{35}$ is used. It is obtained from the full MHD model through a high-aspect-ratio and low- $\beta$ expansion: $\epsilon=a / R_{0} \ll 1, \beta \ll 1$ (e.g., Ref. 36). Here, $a$ and $R_{0}$ are, respectively, the minor and major radius of the torus, $\epsilon$ is the inverse aspect ratio, and the plasma beta $\beta$ $=p /\left(B_{0}^{2} / 2 \mu_{0}\right)$, is the ratio of thermodynamic pressure to magnetic pressure.

The high-aspect-ratio ordering and the presence of a strong axial magnetic field $B_{0}$ allow to express the magnetic field in terms of a magnetic flux function $\Psi$ as $\mathbf{B}=B_{0} \hat{\mathbf{z}}$ $+\nabla \Psi \times \hat{\mathbf{z}}$. Here, $\hat{\mathbf{z}}$ is the unit vector in the axial direction and $B_{0}$ is taken to be constant. In the MHD ordering, the singlefluid velocity $\mathbf{V}$ is approximated by the $\mathbf{E} \times \mathbf{B}$ drift velocity $\mathbf{V}_{E}=-\nabla \Phi \times \hat{\mathbf{z}} / B_{0}$, where $\Phi$ is the electrostatic potential (or stream function). In the limit of zero $\beta$ the pressure equation is decoupled and the RMHD equations take the form of a two-field model:

$$
\begin{aligned}
& \partial_{t} \Psi=-\mathbf{B} \cdot \nabla \Phi+\frac{\eta}{\mu_{0}} \nabla_{\perp}^{2} \Psi+E_{z}, \\
& \rho_{\mathrm{m}} \mathrm{d}_{t} \nabla_{\perp}^{2} \Phi=-\frac{1}{\mu_{0}} \mathbf{B} \cdot \nabla \nabla_{\perp}^{2} \Psi+\nu_{\mathrm{m}} \nabla_{\perp}^{2} \nabla_{\perp}^{2} \Phi .
\end{aligned}
$$

Here $\rho_{\mathrm{m}}$ is the mass density (constant due to the assumption of incompressibility), $\eta$ the plasma resistivity and $\nu_{\mathrm{m}}$ is the kinematic viscosity. The time-independent electric field $E_{z}$ satisfies $\nabla E_{z} \times \hat{\mathbf{z}}=0$. The convective derivative is defined as $\mathrm{d}_{t}=\partial_{t}+\mathbf{V}_{\mathrm{E}} \cdot \nabla$.

Cylindrical geometry is chosen, which gives the righthanded set of coordinates $(r, \vartheta, z)$ where $r \in[0, a]$ is the radius, $\vartheta \in[0,2 \pi]$ the poloidal angle and $z \in\left[0,2 \pi R_{0}\right]$ the axial coordinate (related to the toroidal angle $\varphi$ via $\left.z=-R_{0} \varphi\right)$. The Poisson bracket is defined as $[f, g]=(1 / r)$ $\times\left(\partial_{r} f \partial_{\vartheta} g-\partial_{r} g \partial_{\vartheta} f\right)$, and the Laplacian is approximated by $\nabla^{2} \approx \nabla_{\perp}^{2}=(1 / r) \partial_{r} r \partial_{r}+\left(1 / r^{2}\right) \partial_{\vartheta}^{2}$. In this ordering, toroidal topology is preserved by retaining periodicity in the axial coordinate $z$ and the poloidal angle $\vartheta$.

Normalizing the time by the poloidal Alfvén time $\tau_{\mathrm{Hp}}$ $=\sqrt{\mu_{0} \rho_{\mathrm{m}}} a / B_{\vartheta}(a)$, the radial coordinate by $a$, and introducing the angular coordinate $\zeta \equiv z / R_{0} q(a)$, the normalized variables are $\psi=\Psi / a B_{\vartheta}(a)$ and $\phi=\Phi /\left(a^{2} / \tau_{\mathrm{Hp}}\right)$. Note that $\zeta$ is related to the toroidal angle $\varphi$ via $\zeta=-\varphi / q(a)$, with $q(a)$ $=a B_{0} / R_{0} B_{\vartheta}(a)$ being the safety factor at $r=a$ [cf. Eq. (5)]. The normalization for the source term is $E_{\zeta}$ $=E_{z} /\left[\eta_{0} B_{\vartheta}(a) / \mu_{0} a\right]$. With these normalizations Eqs. (1) and (2) become

$$
\begin{aligned}
& \partial_{t} \psi=[\psi, \phi]-\partial_{\zeta} \phi-S_{\mathrm{Hp}}^{-1}\left(\hat{\eta} j-E_{\zeta}\right), \\
& \partial_{t} u=[u, \phi]+[j, \psi]+\partial_{\zeta} j+R e_{\mathrm{Hp}}^{-1} \nabla_{\perp}^{2} u,
\end{aligned}
$$

where the current density $j$ and the vorticity $u$ are related to $\psi$ and $\phi$ through $j=-\nabla_{\perp}^{2} \psi$ and $u=\nabla_{\perp}^{2} \phi$, respectively. The magnetic Reynolds number $S_{\mathrm{Hp}}$ (also called Lundquist number) and the kinematic Reynolds number $R e_{\mathrm{Hp}}$ are defined as $S_{\mathrm{Hp}} \equiv \tau_{\eta} / \tau_{\mathrm{Hp}}$ and $R e_{\mathrm{Hp}}=a^{2} /\left(\nu_{\mathrm{m}} \tau_{\mathrm{Hp}} / \rho_{\mathrm{m}}\right)$, respectively. Here, $\tau_{\eta}=a^{2} \mu_{0} / \eta(r=0)$ is the resistive diffusion time. The resistivity profile is given by $\hat{\eta}(r)$, normalized such that $\hat{\eta}(r=0)$ $=1$. The relative strengths of kinetic viscosity and (resistive) diffusion are characterized by the Prandtl number $\mathrm{Pr}$ $=S_{\mathrm{Hp}} / R e_{\mathrm{Hp}} \propto \nu / \eta$, with $\nu=\nu_{\mathrm{m}} / \rho_{\mathrm{m}}$ being the specific ion viscosity.

\section{B. RMHD equilibrium and resonant modes}

The equilibrium state is defined as $\partial_{t} \psi=\partial_{t} \phi=0$. Since the effect of the plasma pressure is neglected, the structure of the equilibrium magnetic field $\mathbf{B}_{\text {eq }}$ is given in terms of the tokamak safety factor $q$, defined as

$$
q=\frac{\mathbf{B}_{\mathrm{eq}} \cdot \nabla \zeta}{\mathbf{B}_{\mathrm{eq}} \cdot \nabla \vartheta} .
$$

The safety factor measures the field line pitch by counting how many times a magnetic field line goes the long way around the torus $\left(2 \pi R_{0}\right)$ after one turn the short way around $(2 \pi r)$. Here, $q=q(r)$, so that the equilibrium magnetic flux surfaces, where $\psi=$ const, are uniquely defined by the radius $r$. Each field variable $f$ is written in terms of a timeindependent equilibrium component $\bar{f}$ and a time-dependent perturbation $\tilde{f}$ as

$$
f(r, \vartheta, \zeta, t)=\bar{f}(r)+\widetilde{f}(r, \vartheta, \zeta, t) .
$$

It is assumed that the equilibrium state is free of flows,

$$
\bar{\phi}=\bar{u}=0 .
$$



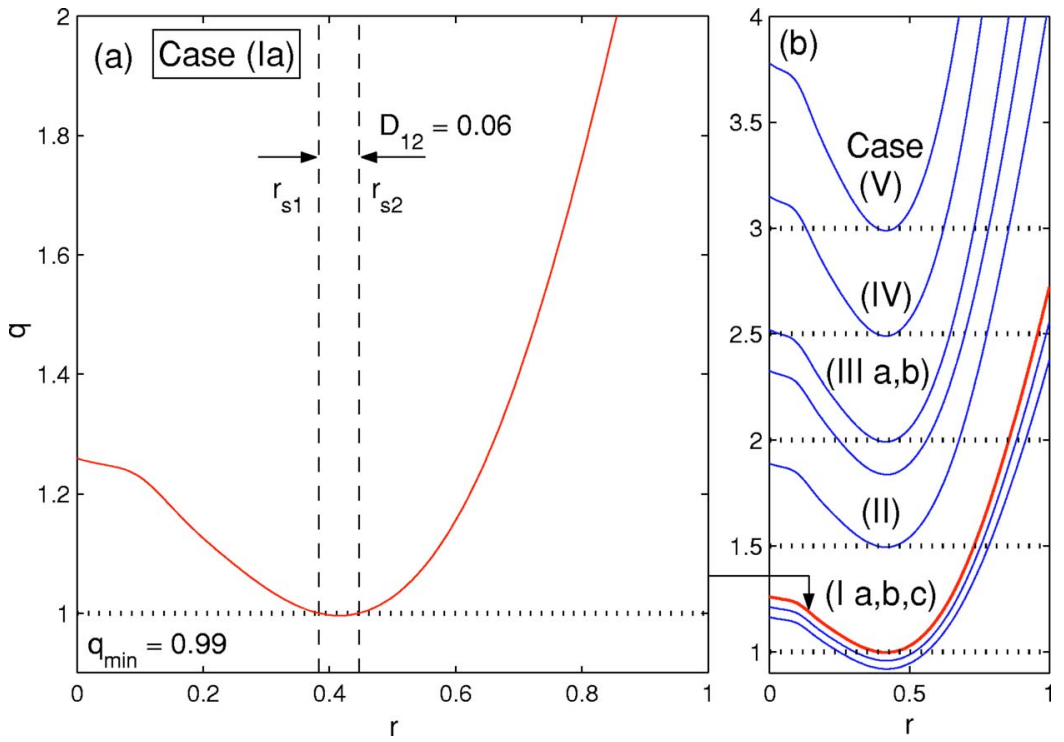

FIG. 1. (Color online). Safety factor profiles with two resonant surfaces used in this study. The Case (Ia), plotted in (a), has two $q_{\mathrm{s}}=1$ resonances, located at $r=r_{\mathrm{s} 1}$ and $r_{\mathrm{s} 2}$, a relatively small distance $D_{12}=0.06$ apart. In (b), variants of this profile, with different interresonance distances $D_{12}$ and different $q_{\mathrm{s}}$ are shown. The parameter values required to reproduce Case (Ia) are given in Table I and the geometric characteristics of all cases are listed in Table II.
In general, a tokamak plasma has magnetic surfaces where $q_{\mathrm{s}}=q\left(r_{\mathrm{s}}\right)=m / n$, with integers $m$ and $n$. These are called rational or resonant surfaces. The radius $r_{\mathrm{s}}$ is called resonant radius, because an infinitesimally small helical magnetic perturbation with helicity $h=m / n$ is resonant with the magnetic field structure in the vicinity of $r_{\mathrm{s}}$. Such a resonant perturbation $\delta \tilde{\psi}_{m, n}$ does not bend field lines, so there is no restoring force. If the perturbation leads the system to a state of lower energy, the amplitude of the resonant perturbation will grow and the mode is said to be unstable.

\section{Fourier representation}

In order to study the properties of such resonant modes it is useful to apply a Fourier transform with respect to the periodic coordinates: $(\vartheta, \zeta) \rightarrow(m, n)$. This representation also gives an efficient and accurate numerical model. Substituting for each field variable $f$ in Eqs. (3) and (4) the Fourier expansion

$$
f(r, \vartheta, \zeta, t)=\frac{1}{2} \sum_{m, n} f_{m, n}(r, t) \cdot e^{i(m \vartheta-n \zeta)}+\text { c.c. },
$$

one obtains equations for the individual Fourier modes,

$$
\begin{aligned}
& \partial_{t} \psi_{m, n}=[\psi, \phi]_{m, n}+i n \phi_{m, n}-S_{\mathrm{Hp}}^{-1}\left(\hat{\eta} j_{m, n}-E_{m, n}\right), \\
& \partial_{t} u_{m, n}=[u, \phi]_{m, n}+[j, \psi]_{m, n}-i n j_{m, n}+R e_{\mathrm{Hp}}^{-1} \nabla_{m, n}^{2} u_{m, n},
\end{aligned}
$$

where $\nabla_{m, n}^{2}=(1 / r) \partial_{r} r \partial_{r}-m^{2} / r^{2}$ and the nonlinear terms $[f, g]_{m, n}$ have acquired the form of convolutions. In this model, if a perturbation is applied only to modes of a given helicity $h=m / n$, modes with different helicities will not be excited and the problem is effectively reduced to a two- dimensional one. In the following, we will exclusively refer to individual Fourier components of the field variables and usually omit the $(m, n)$ subscripts for convenience. Note that the equilibrium fields $\bar{f}=\bar{f}(r)$ have only $(m, n)=(0,0)$ components.

\section{Equilibrium model}

In order to study configurations with two resonant surfaces, the following model formula for the equilibrium $q$ profile is used:

$$
q(r)=q_{0} \cdot F_{1}(r) \cdot\left\{1+\left(r / r_{0}\right)^{2 \mu(r)}\right\}^{1 / \mu(r)},
$$

where

$$
\begin{aligned}
& r_{0}=r_{\mathrm{A}}\left|\left[m /\left(n q_{0}\right)\right]^{\mu\left(r_{\mathrm{A}}\right)}-1\right|^{-1 / 2 \mu\left(r_{\mathrm{A}}\right),} \\
& \mu(r)=\mu_{0}+\mu_{1} r^{2}, \\
& F_{1}(r)=1+f_{1} \exp \left\{-\left[\left(r-r_{11}\right) / r_{12}\right]^{2}\right\} .
\end{aligned}
$$

The parameter set $\left\{q_{0}, r_{\mathrm{A}}, \mu_{0}, \mu_{1} m, n\right\}$ is used to design the underlying monotonic profile. The parameters $\left\{f_{1}, r_{11}, r_{12}\right\}$ describe the Gaussian bump which is used to create nonmonotonic profiles with two (or three) $q_{\mathrm{s}}=\mathrm{m} / \mathrm{n}$ resonant surfaces. The DTM configurations used in this study are shown in Fig. 1. The corresponding model parameters are listed in Table I and the geometric characteristics of all cases are given in Table II.

With $q$ given by Eq. (11), $\bar{\psi}$ and $\bar{j}$ are calculated using the relations

TABLE I. Parameter values for Eq. (11), giving the $q$ profile shown in Fig. 1(a). The other cases are readily obtained by changing $m$ and $n$ (so that $q$ s $=m / n)$ and $q_{0}$.

\begin{tabular}{ccccccccc}
\hline \hline$q_{0}$ & $r_{\mathrm{A}}$ & $\mu_{0}$ & $\mu_{1}$ & $m$ & $n$ & $f_{1}$ & $r_{11}$ \\
\hline 1.3 & 0.655 & 3.8824 & 0 & 1 & 1 & -0.238 & 0.4286 & 0.304 \\
\hline \hline
\end{tabular}


TABLE II. Geometric properties of the $q$ profiles shown in Fig. 1(b).

\begin{tabular}{cccccc}
\hline \hline Case & $q_{\mathrm{s}}$ & $q_{\min }$ & $D_{12}$ & $s_{1}$ & $s_{2}$ \\
\hline (Ia) & 1 & 0.99 & 0.06 & -0.10 & 0.12 \\
$(\mathrm{Ib})$ & 1 & 0.96 & 0.21 & -0.20 & 0.45 \\
$(\mathrm{Ic})$ & 1 & 0.92 & 0.31 & -0.20 & 0.66 \\
$(\mathrm{II})$ & $3 / 2$ & 1.49 & 0.06 & -0.10 & 0.12 \\
$(\mathrm{III})$ & 2 & 1.92 & 0.31 & -0.20 & 0.66 \\
(IIIb) & 2 & 1.99 & 0.06 & -0.10 & 0.12 \\
(IV) & $5 / 2$ & 2.49 & 0.06 & -0.10 & 0.12 \\
(V) & 3 & 2.99 & 0.06 & -0.10 & 0.12 \\
\hline \hline
\end{tabular}

$$
q^{-1}=-\frac{1}{r} \frac{\mathrm{d} \bar{\psi}}{\mathrm{d} r} \quad \text { and } \quad \bar{j}=-\nabla_{\perp}^{2} \bar{\psi}
$$

The resonant surfaces where $q=q_{\mathrm{s}}$ are labeled with $r_{\mathrm{s} 1}$ and $r_{\mathrm{s} 2}$, and the inter-resonance distance is given by $D_{12}=\mid r_{\mathrm{s} 2}$ $-r_{\mathrm{s} 1} \mid$. The magnetic shear profile is defined as

$$
s=\frac{r}{q} \frac{\mathrm{d} q}{\mathrm{~d} r}=\frac{\mathrm{d}(\ln q)}{\mathrm{d}(\ln r)},
$$

and the local magnetic shear at the resonant radius $r_{\mathrm{s} i}$ is $s_{i}$ $=s\left(r_{\mathrm{si}}\right)$.

The time-independent source term $E_{\zeta}$ compensates the resistive dissipation of the equilibrium current profile, i.e., $E_{\zeta}=\hat{\eta} \bar{j}$ (diffusive equilibrium). For numerical simulations where the temporal evolution of the resistivity profile $\hat{\eta}$ is neglected one often assumes $\hat{\eta}(r)=\bar{j}(r=0) / \bar{j}(r)$. This will generally lead to different values of the resistivity at different $r_{\mathrm{s} i}$. In order to simplify the comparison between growth rates of modes associated with different resonant surfaces, a homogeneous resistivity profile, $\hat{\eta}(r)=1$, is used in this study. Numerical tests indicate that the details of $\hat{\eta}$ have no significant effect on any of the qualitative characteristics discussed in this paper.

\section{E. Linearized equations}

When the amplitudes and gradients of the perturbed fields are sufficiently small the nonlinear terms in Eqs. (9) and (10) may be neglected and one obtains the linearized RMHD equations. In the linear system, the time dependence of a perturbed field variable $\tilde{f}$ takes the form

$$
\tilde{f}(r, t)=f(r) \cdot \exp (\lambda t),
$$

where $\lambda$ is a complex number. Using Eq. (14), the system of equations (9) and (10) becomes

$$
\begin{aligned}
\lambda \tilde{\psi}= & i\left(n-\frac{m}{q}\right) \widetilde{\phi}+\frac{1}{S_{\mathrm{Hp}}}\left(\frac{1}{r} \partial_{r} r \partial_{r}-\frac{m^{2}}{r^{2}}\right) \tilde{\psi} \\
\lambda \tilde{u}= & i\left(n-\frac{m}{q}\right)\left(\frac{1}{r} \partial_{r} r \partial_{r}-\frac{m^{2}}{r^{2}}\right) \tilde{\psi}+\frac{i m}{r}\left(\frac{s(s-2)}{r q}-\frac{\partial_{r} s}{q}\right) \tilde{\psi} \\
& +\frac{1}{R e_{\mathrm{Hp}}}\left(\frac{1}{r} \partial_{r} r \partial_{r}-\frac{m^{2}}{r^{2}}\right) \tilde{u} .
\end{aligned}
$$

Equations (15) and (16) are obtained by applying Eqs. (12) and (13) and expressing the equilibrium fields in terms of the safety factor $q=q(r)$ and the magnetic shear $s=s(r)$.

Modes for which the linear growth rate is positive, $\gamma_{\text {lin }}$ $=\mathfrak{R}\{\lambda\}>0$, are said to be linearly unstable. Their amplitudes grow exponentially in time, e.g.,

$$
\delta \tilde{\psi}_{m, n}(r, t)=\delta \tilde{\psi}_{m, n}(r) \exp \left(\gamma_{\text {lin }} t\right)
$$

(in RMHD, $\mathfrak{I}\{\lambda\}=0$ ). The linear growth rate $\gamma_{\text {lin }}$ is a function of the mode numbers,

$$
\gamma_{\text {lin }}=\gamma_{\text {lin }}(m, n)
$$

(spectrum of growth rates), and written this way it is generally referred to as the dispersion relation. Since this study is restricted to modes with unique helicity, $h$, it is sufficient to specify $m$ for a given $q_{\mathrm{s}}=h\left(\right.$ so $\left.n=m / q_{\mathrm{s}}\right)$. Along with the radial structure of the eigenmodes, $\widetilde{\psi}(r)$ and $\widetilde{\phi}(r)$, the linear

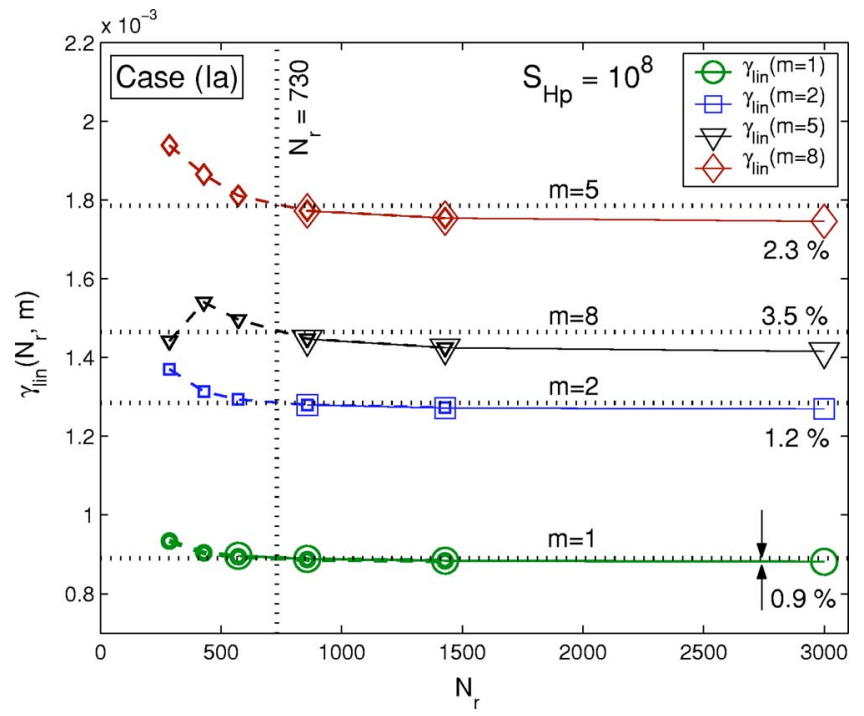

FIG. 2. (Color online). Numerical convergence of the linear growth rates $\gamma_{\text {lin }}(m)$ with increasing $N_{r}$ (number of radial grid points per unit length). The configuration used is Case (Ia) with $D_{12}=0.06, S_{\mathrm{Hp}}=10^{8}$ and $R e_{\mathrm{Hp}}=10^{12}$. The growth rates are shown for modes $m=1,2,5$, and 8 . The $m=5$ mode is dominant in this case. The small symbols represent EVP results, calculated in the range $250 \leqslant N_{r} \leqslant 1430$. The large symbols represent IVP results. The IVP solver was run with up to $N_{r}=3000$. The agreement between both methods and the numerical convergence is clearly shown. The horizontal dotted lines indicate the linear growth rate values for $N_{r}=730$. This is the standard $N_{r}$ value used for $S_{\mathrm{Hp}}=10^{8}$ in this paper (cf. Table III). It can be seen that these growth rates differ from the $N_{r}=3000$ results only by a few percent. This accuracy is sufficient for the purpose of this paper. 
TABLE III. Typical values for $N_{r}$ (number of radial grid points per unit length) used in the EVP solver. The IVP code is usually run with $2000 \leqq N_{r} \leqslant 3000$.

\begin{tabular}{ccccc}
\hline \hline$S_{\mathrm{Hp}}:$ & $\$ 10^{5}$ & $10^{5} \rightarrow 10^{7}$ & $10^{7} \rightarrow 10^{8}$ & $>10^{8}$ \\
$N_{r}:$ & $180-360$ & $430-570$ & 730 & 1460 \\
\hline \hline
\end{tabular}

growth rates are our main tool for characterizing the linear instability of DTMs under various conditions.

\section{F. Numerical method}

After discretizing Eqs. (15) and (16) with respect to the radial coordinate, the linearized RMHD model may be written as a generalized eigenvalue problem,

$$
\mathbf{B} \Lambda \mathbf{x}=\mathbf{A} \mathbf{x},
$$

where $\mathbf{x}^{\mathrm{T}}=[\tilde{\psi}, \widetilde{\phi}], \boldsymbol{\Lambda}$ is a diagonal matrix containing the eigenvalues $\lambda$, and $\mathbf{A}$ and $\mathbf{B}$ are coefficient matrices, which also include the finite-difference operators. All eigenmodes $\mathbf{x}_{j}$ with corresponding eigenvalues $\lambda_{j}$ are obtained using an eigenvalue problem (EVP) solver. The accuracy of the results is checked by running tests with different numbers of radial grid points and through comparison with results obtained by solving the linearized version of Eqs. (1) and (2) as an initial value problem (IVP). The numerical convergence and the agreement between the EVP and IVP results are demonstrated in Fig. 2. In Table III the radial resolution used for different values of $S_{\mathrm{Hp}}$ is specified. Due to numerical constraints, the regime $S_{\mathrm{Hp}}>10^{10}$ was not accessible with sufficient accuracy.

\section{RESULTS}

\section{A. Mode structures}

The radial structure of an eigenmode determines which part of the plasma is affected by the instability, since $\phi(r)$ is related to the radial and poloidal components of the plasma displacement velocity,

$$
v_{r}=-\frac{i m}{r} \phi \quad \text { and } \quad v_{\vartheta}=\partial_{r} \phi
$$

In general, for each $(m, n)$ there may be as many unstable eigenmodes as there are resonant surfaces $q_{\mathrm{s}}=m / n$. The radial mode structures of unstable $q_{\mathrm{s}}=1$ DTMs are shown in Fig. 3. The configuration used is Case (Ia), where $D_{12}=0.06$. For $m=1$ [Figs. 3(a) and 3(c)] there are two unstable eigenmodes. For $m>1$ [Figs. 3(b) and 3(d)] only one eigenmode is unstable (the $m=8$ mode structure shown is representative for other modes with $m>1$ ). In our notation, the eigenmode $M^{(1)}$ is associated with the innermost resonant surface $r_{\mathrm{s} 1}$. For $m=1$ it has a finite amplitude in the region $0<r<r_{\mathrm{s} 1}$. The eigenmode $M^{(2)}$ is active in the region 0 $<r<r_{\mathrm{s} 2}$ for $m=1$, and mainly in the region $r_{\mathrm{s} 1}<0<r_{\mathrm{s} 2}$ for $m>1$.

In Fig. 4, the mode structures of $q_{\mathrm{s}}=2$ DTMs with $(m, n)=(2,1)$ are plotted. Results are shown for two cases with different distances $D_{12}$ : Case (IIIa) with $D_{12}=0.31$
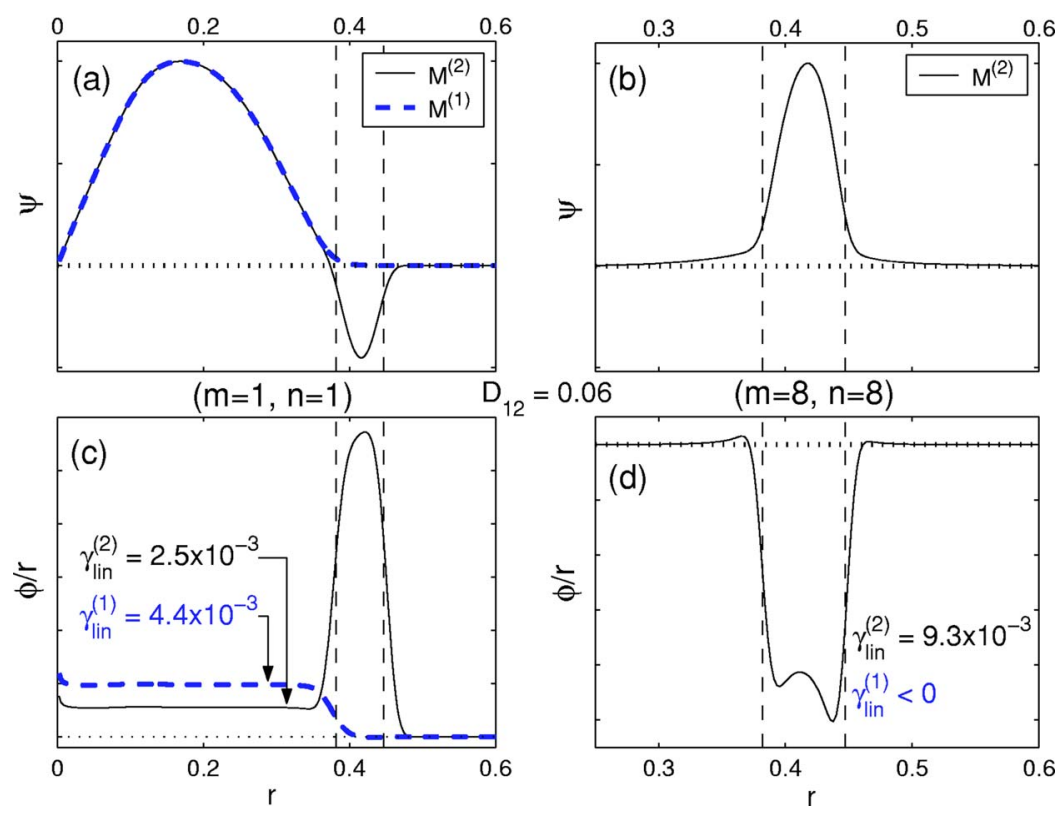

FIG. 3. (Color online). Mode structures of the unstable $q_{\mathrm{s}}=1$ DTM eigenmodes of Case (Ia), where $D_{12}=0.06$. The locations of the resonant surfaces are indicated by vertical dashed lines. Diagrams (a) and (c) show, respectively, $\psi$ and $\phi / r$ of the $m=1$ mode. In (b) and (d) the mode structures for $m=8$ are shown, which are representative for other modes with $m>1$. In our notation, the eigenmode $M^{(1)}$ extends to $r=r_{\mathrm{s} 1}$, and $M^{(2)}$ to $r_{\mathrm{s} 2}$. These results are obtained with $S_{\mathrm{Hp}}=10^{6}$, but similar mode structures are also found for other values of $S_{\mathrm{Hp}}$ $>10^{5}$. The linear growth rates $\gamma_{\operatorname{lin}}^{(1)}$ and $\gamma_{\text {lin }}^{(2)}$ are also shown in (b) and (d). 

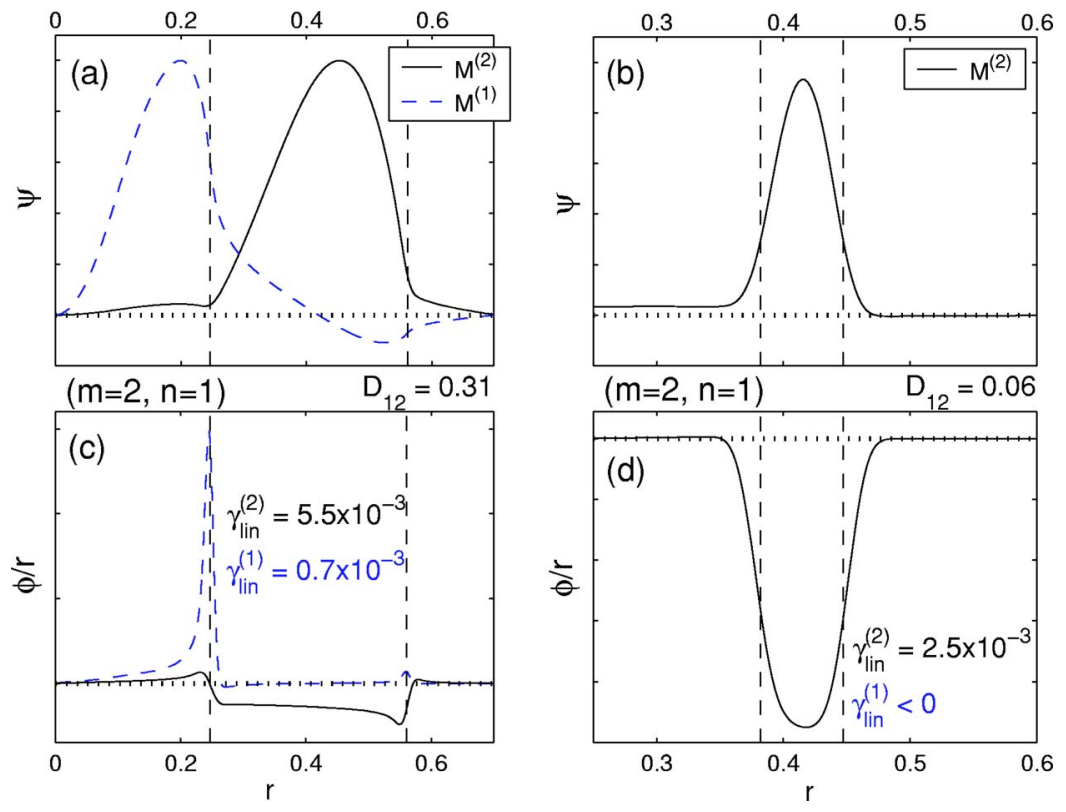

FIG. 4. (Color online). Mode structures of the unstable $q_{\mathrm{s}}=2$ DTM eigenmodes with $(m, n)=(2,1)$. In (a) and (c), respectively, $\psi$ and $\phi / r$ are shown for Case (IIIa) $\left(D_{12}=0.31\right)$. In (b) and (d) the results for Case (IIIb) $\left(D_{12}=0.06\right)$ are plotted. These results were obtained with $S_{\mathrm{Hp}}=10^{6}$.
[Figs. 4(a) and 4(c)], and Case (IIIb) with $D_{12}=0.06$ [Figs. 4(b) and 4(d)]. In agreement with previous works on DTMs, it is found that two individual modes are present in the case of larger $D_{12}: M^{(1)}$ with even parity around $r_{\mathrm{s} 1}$ and $M^{(2)}$ with odd parity. ${ }^{37,28}$ In the case of smaller $D_{12}$ only the eigenmode $M^{(2)}$ is found to be unstable.

The $M^{(1)}$ eigenmode is essentially an STM associated with the $r=r_{\mathrm{s} 1}$ resonant surface (here, a negative-shear surface), since it is practically unaffected by the presence of resonant surfaces beyond $r=r_{\mathrm{s} 1}$. The actual DTMs (for sufficiently small $\left.D_{12}\right)$ are the $M^{(2)}$ eigenmodes. The radial structure of DTMs with $m>1$ is very similar for different $q_{\mathrm{s}}$, as may be seen by comparing the profiles (b) and (d) in Figs. 3 and 4. Let us note that Cases (II), (IV), and (V) of Table II, where $q_{\mathrm{s}}=3 / 2,5 / 2$ and 3 , respectively, have eigenmode structures very similar to those found for $q_{\mathrm{s}}=2$ in Fig. 4.

The local resistivity $\eta\left(r_{s i}\right)$ and the magnetic shear $s\left(r_{s i}\right)$ at a resonant surface $r_{s i}$, together with the distance between neighboring resonant surfaces $D_{12}$, determine which eigenmode, $M^{(1)}$ or $M^{(2)}$, will be the dominant mode for a given $m$. An exception is the STM-like eigenmode $M^{(1)}$ : as its mode structure indicates, it is not affected by $D_{12}$.

\section{B. Viscosity effect}

Although, it is difficult to obtain reliable values for the ion viscosity in the tokamak core, it is usually thought to be very low. However, it is required in most nonlinear simulations for the purpose of providing a cut-off at short wavelengths in order to be consistent with the finite number of grid points or Fourier modes. As may be seen from Fig. 5, as long as the Prandtl number satisfies $P r \lesssim 0.1$, the viscosity has practically no effect on the linear growth rates of DTMs. A similar result is also obtained for other values of $S_{\mathrm{Hp}}$ as well as for single and triple tearing modes, so it may be considered a generic characteristic of tearing modes. The mode structures in Figs. 3 and 4 and all the following results were obtained in the regime where $\operatorname{Pr}<0.01$, so the viscosity effect will not be discussed further in this paper. Let us note that the stabilizing effect of viscosity for $\operatorname{Pr} \gtrsim 1$, evident in Fig. 5, was pointed out previously by $\mathrm{Ofman}^{31}$ in a study on DTMs in the presence of equilibrium shear flows.

\section{Dispersion relations for $q_{s} \geqslant 1$}

Recently it was discovered that configurations with nearby $q_{\mathrm{s}}=1$ resonant surfaces have broad spectra of linearly unstable modes. ${ }^{4}$ In particular, if the distance between the resonant radii is sufficiently small, modes with $m \sim \mathcal{O}(10)$ were found to have linear growth rates several times higher than the $m=1$ mode.

Indeed, similar behavior is observed when the resonances are located at higher $q$ values, such as $q_{\mathrm{s}}=3 / 2,2$, 5/2, and 3, corresponding to Cases (IIb), (III), (IV) and (V), respectively. The dispersion relations for these cases are

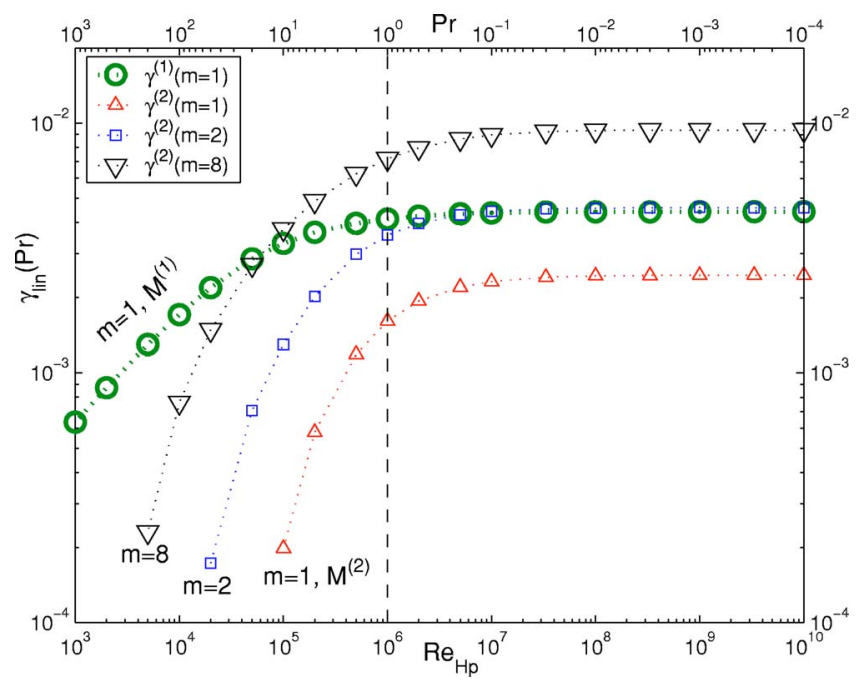

FIG. 5. (Color online). Dependence of the linear DTM growth rates on the Prandtl number [Case (Ia)]. The growth rates of eigenmodes with $m$ $=1,2,8$ are plotted for fixed $S_{\mathrm{Hp}}=10^{6}$. 

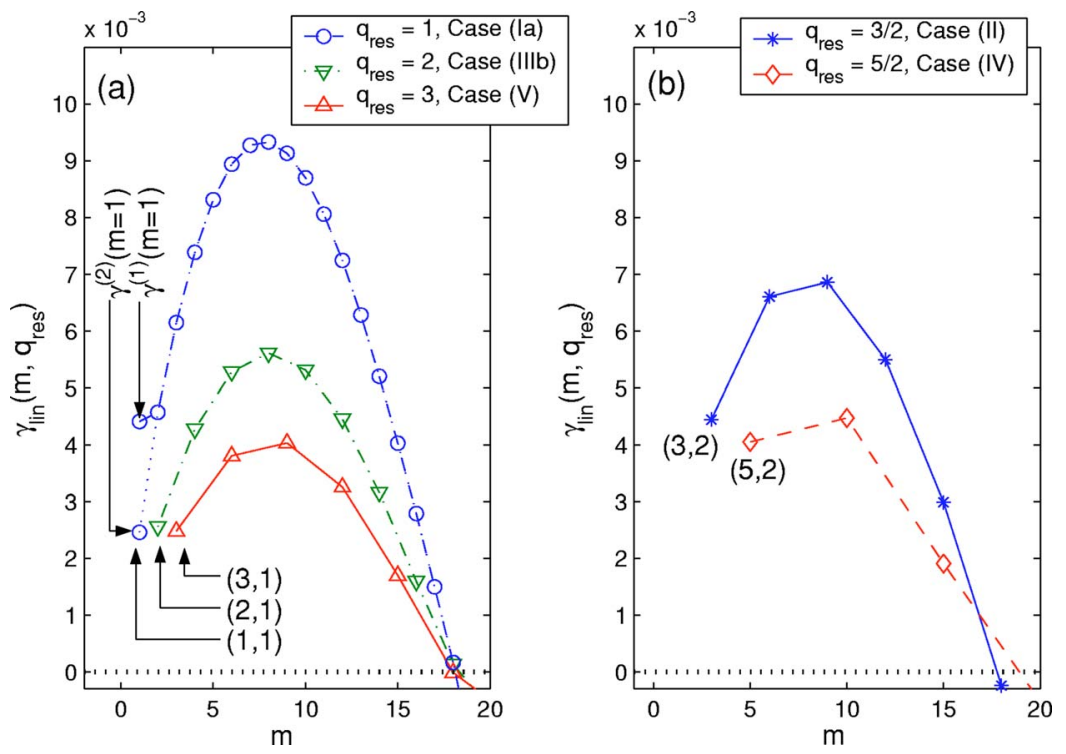

FIG. 6. (Color online). Comparison between dispersion relations for (a) $q_{\mathrm{s}}=1,2$ and 3 , and (b) $q_{\mathrm{s}}=3 / 2$ and $5 / 2$. For all cases $D_{12}=0.06$ (cf. Table II). For $m=1$, circles in diagram (a), growth rates of both unstable modes are shown: $\gamma_{\text {lin }}^{(1)}=4.1 \times 10^{-3}, \quad \gamma_{\text {lin }}^{(2)}=2.6 \times 10^{-3}$. All other growth rates belong to $M^{(2)}$-type eigenmodes, since the $M^{(1)}$-type modes are stable for $m>1$ in these cases. For all cases $S_{\mathrm{Hp}}=10^{6}$.

shown in Fig. 6. All these $q$ profiles have the same values for $D_{12}, s_{1}$ and $s_{2}$. They only differ from each other in the values of $q_{\min }$ and $q_{\mathrm{s}}$. Let us remark that for all cases the width of the spectrum as well as the location of the maximum growth rate is practically identical: $\gamma_{\text {lin }}>0$ for $1 \leqslant m \leqslant 18$, and the peak

$$
\gamma_{\text {peak }}=\gamma_{\text {lin }}\left(m_{\text {peak }}\right)=\max \left\{\gamma_{\text {lin }}(m)\right\}
$$

is located at $m=8$. Except for the $m=1$ mode $\left[q_{\mathrm{s}}=1\right.$, Case (Ia)], all growth rates in Fig. 6 are associated with $M^{(2)}$-type eigenmodes. Due to these results it may be conjectured that all DTMs [except for $M^{(2)}(m=1)$ ] behave similarly for any $q_{\mathrm{s}}$, including $q_{\mathrm{s}}=1$.

\section{Role of $D_{12}$}

Consider the scenario where $q_{\text {min }}$ gradually drops below $q_{\mathrm{s}}=1$ due to an increase in the current density. During this process the distance between the resonant surfaces will grow in time, starting from $D_{12}=0$ when $q_{\text {min }}=q_{\mathrm{s}}$. The series of
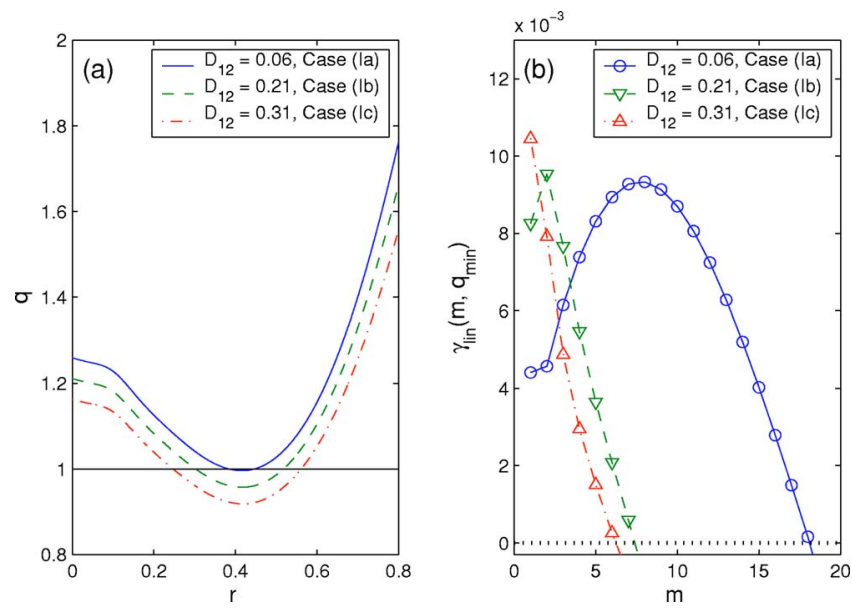

FIG. 7. (Color online). Role of $D_{12}$ (variable shears) for the instability of $q_{\mathrm{s}}=1$ DTMs. In (a) the $q$ profiles are shown [Cases (Ia,b,c) in Table II] and in (b) the corresponding dispersion relations are plotted. For all cases $S_{\mathrm{Hp}}$ $=10^{6}$, and only the growth rate of the dominant mode is shown for each $m$.
Cases (Ia), (Ib) and (Ic), shown in Fig. 7(a), represent the equilibria at successive instants in time during such a process. Similar cases were previously investigated in Ref. 28 where the effect of $D_{12}$ on $\gamma_{\text {lin }}(m=3, n=1)$ and the corresponding scaling exponent $\alpha$ (in $\gamma_{\text {lin }} \propto S_{\mathrm{Hp}}^{-\alpha}$ ) was characterized. In Ref. 38 , the role of $q_{\text {min }}$ was studied in the context of partial and full reconnection.

The dispersion relations for the three cases of Fig. 7(a) are plotted in Fig. 7(b). Clearly, the width of the spectrum is reduced as $D_{12}$ increases, and the $m=1$ mode eventually becomes dominant.

Note that Cases (Ia)-(Ic) all have different magnetic shears $s_{1}$ and $s_{2}$ [cf. Table II]. The effect of varying only $D_{12}$ is illustrated in Fig. 8. Starting with Case (Ib), where $D_{12}$ $=0.21$, the $q$ profile is gradually modified in such a way that $D_{12}$ decreases down to 0.08 , while both shears, $s_{1}$ and $s_{2}$, are
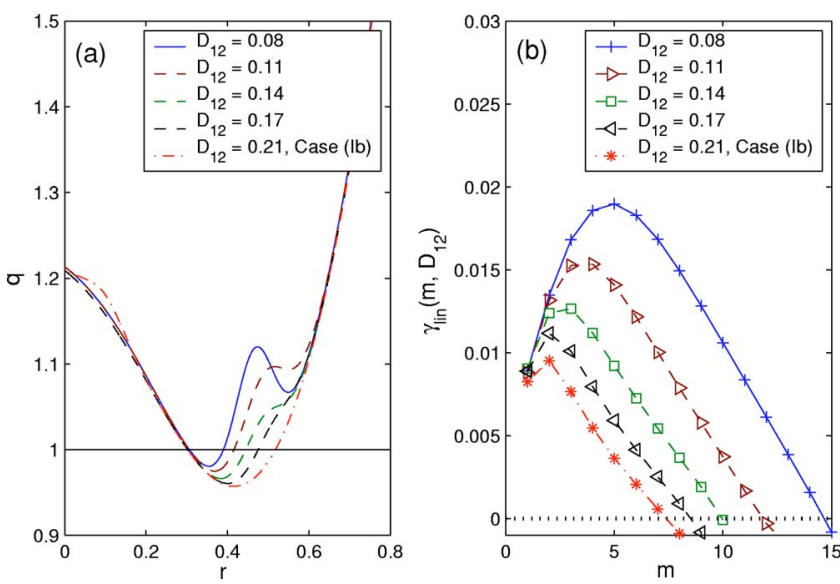

FIG. 8. (Color online). Role of $D_{12}$ (constant shears) for the instability of $q_{\mathrm{s}}=1$ DTMs. In (a) the $q$ profiles used are shown. The basic profile is Case (Ib), with $D_{12}=0.21$. The other profiles $\left(D_{12} \leqslant 0.17\right)$ were obtained by gradually reducing $D_{12}$, but holding the shears $s_{1}=-0.20$ and $s_{2}=0.45$ constant. For this, the model equation (11) was multiplied by another factor, $F_{2}(r)$, defined equivalently to $F_{1}(r)$. In (b) the corresponding dispersion relations are shown. For all cases $S_{\mathrm{Hp}}=10^{6}$, and only the growth rate of the dominant mode is shown for each $m$. 

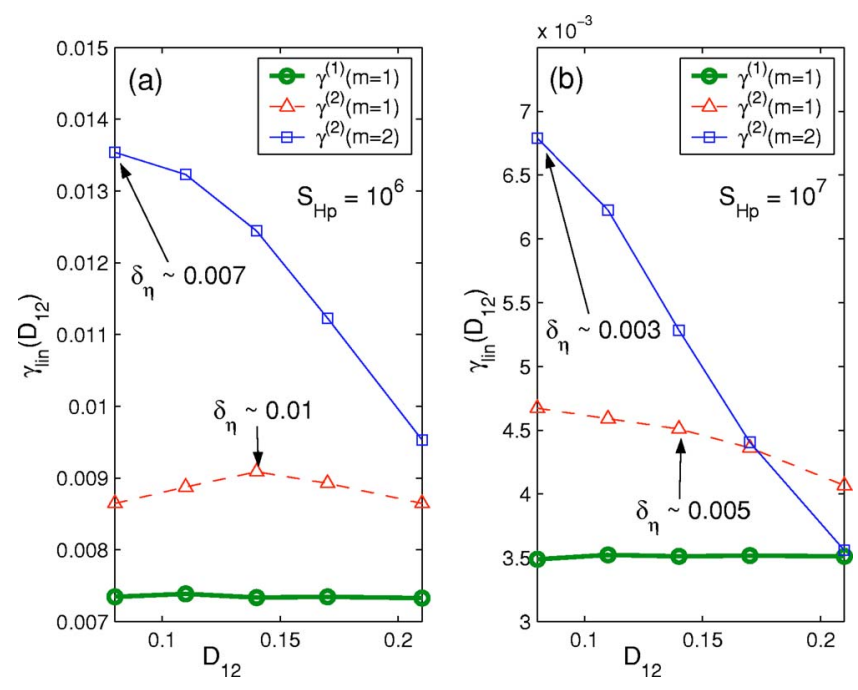

FIG. 9. (Color online). $D_{12}$ dependence of the growth rates of $m=1$ and $m=2$ DTMs with $q_{\mathrm{s}}=1$. While varying $D_{12}$, the shears $s_{1}=-0.20$ and $s_{2}$ $=0.45$ were held constant. The values for the Lundquist number are (a) $S_{\mathrm{Hp}}=10^{6}$ and (b) $S_{\mathrm{Hp}}=10^{7}$. For several data points, indicated by arrows, estimates are given for the linear resistive layer width $\delta_{\eta}$ defined by Eq. (22).

held constant. The profiles used are plotted in Fig. 8(a) and the corresponding dispersion relations in Fig. 8(b). These results show that $D_{12}$ controls the broadness of the spectrum. Modes with higher $m$ and with higher growth rates than the lower- $m$ modes appear when $D_{12}$ is decreased.

It is known that a tearing mode is stable when the local magnetic shear at the resonant surface is zero. ${ }^{26}$ The results in Fig. 7 indicate that for DTMs with $m>1$ the destabilizing effect of small $D_{12}$ dominates over the effect of low local shears $\left[\left|s_{1}\right|\right.$ and $s_{2}$ become small when $\Delta q=q_{\mathrm{s}}-q_{\min }$ is reduced (cf. Table II)].

The dependence of the DTM growth rates on the parameter $q_{\text {min }}$ (meaning, simultaneous variation of $D_{12}, s_{1}$, and $s_{2}$ ) was studied previously by Ishii et al. ${ }^{28}$ for the $(m, n)$ $=(3,1)$ mode and a set of $q$ profiles similar to those in Fig. $7(\mathrm{a})$. They found that the curve $\gamma_{\operatorname{lin}}\left(q_{\min }\right)$ is not monotonic. In Fig. 9(a) it is shown that this is also true for the dependence of $\gamma_{\text {lin }}$ on $D_{12}$ alone (constant shears): in the range shown $\left(0.08 \leqslant D_{12} \leqslant 0.21\right)$, the growth rate of the $M^{(2)}(m$ =1) mode, $\gamma_{\operatorname{lin}}^{(2)}(m=1)$, has a maximum around $D_{12}=0.14$. A comparison between the results for $S_{\mathrm{Hp}}=10^{6}$ in Fig. 9(a) and $S_{\mathrm{Hp}}=10^{7}$ in Fig. 9(b) shows that the value $S_{\mathrm{Hp}}$ influences the location of the peak. This is most likely related to the distance $D_{12}$ becoming comparable to the linear resistive layer width $\delta_{\eta}$, which is estimated after Ref. 2,

$$
\delta_{\eta} \simeq\left[\frac{\gamma_{\mathrm{lin}}(m)}{\left(m / r_{\min }\right)^{2} B_{\mathrm{s}}^{\prime 2} S_{\mathrm{Hp}}}\right]^{1 / 4},
$$

where $B_{\mathrm{s}}^{\prime}=s\left(r_{s i}\right) / q\left(r_{s i}\right)$. In the following section, the role of $S_{\mathrm{Hp}}$ will be studied in more detail.

It is noted that the growth rate $\gamma_{\operatorname{lin}}^{(1)}(m=1)$ does not depend on $D_{12}$, as it is expected from the mode structure $M^{(1)}(m=1)$ [Figs. 3(a) and 3(c)]. Hence, the increase of the $m=1$ growth rate in Fig. 7(b) is caused by the increase in the magnetic shear $\left|s_{1}\right|$.

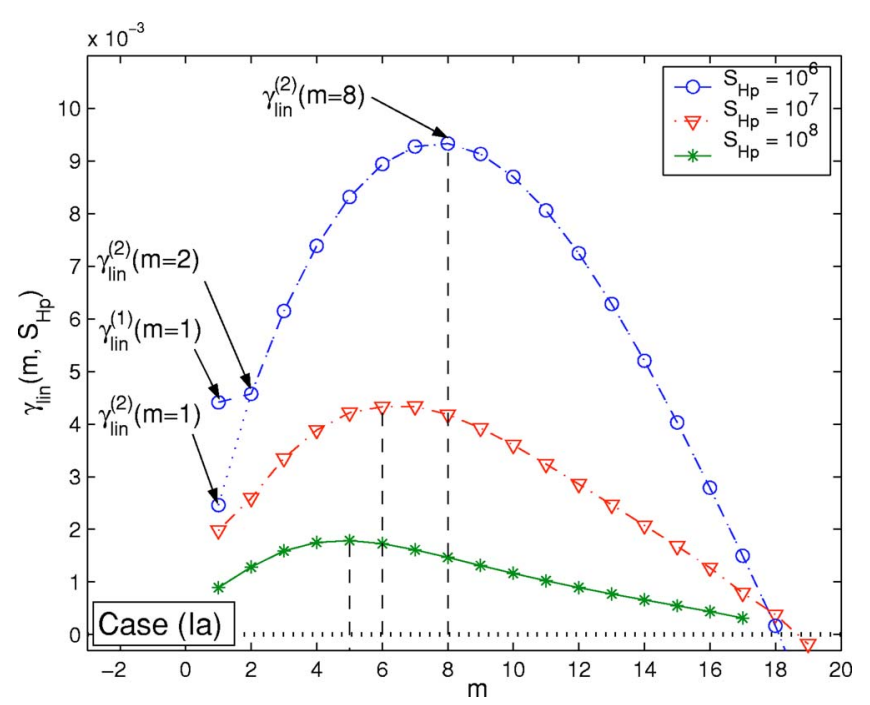

FIG. 10. (Color online). DTM dispersion relations for $S_{\mathrm{Hp}}=10^{6}, 10^{7}$, and $10^{8}$ obtained with Case (Ia) where $q_{\mathrm{s}}=1$. For $m=1$ there are two unstable eigenmodes, $M^{(1)}$ and $M^{(2)}$. The growth rates of both the dominant $\left(M^{(1)}\right)$ and the secondary $\left(M^{(2)}\right) m=1$ eigenmode are shown for $S_{\mathrm{Hp}}=10^{6}$ (they almost coincide for higher $S_{\mathrm{Hp}}$ ). Vertical dashed lines indicate the locations $m_{\text {peak }}$ of the peaks $\gamma_{\text {peak }}=\operatorname{Max}\left\{\gamma_{\text {lin }}(m)\right\}$. Similar results are obtained for cases with $q_{\mathrm{s}}>1$.

\section{E. Role of resistivity}

In the previous sections the linear instability of DTMs was investigated at $S_{\mathrm{Hp}}=10^{6}$, since this value lies in the regime where nonlinear simulations of MHD instabilities are often performed: $10^{4} \leq S_{\mathrm{Hp}} \leqslant 10^{7}$. While large tokamaks typically operate in regimes where $S_{\mathrm{Hp}} \gtrsim 10^{8}$ (except in the early current-ramp-up phase), it is difficult to access this lowcollisional regime with nonlinear simulations. Therefore, the linear study of the $S_{\mathrm{Hp}}$ dependence of DTM growth rates is an important tool for relating nonlinear simulation results to plasma conditions.

In Fig. 10 the dispersion relations of Case (Ia) are shown for $S_{\mathrm{Hp}}=10^{6}, 10^{7}$ and $10^{8}$. The growth rates of all modes are found to decrease with increasing $S_{\mathrm{Hp}}$, as it is expected for resistive instabilities. Let us remark that the width of the spectrum is not affected by the variation of $S_{\mathrm{Hp}}$ : unstable modes are found in the range $1 \leqslant m \leqslant 18$. However, it can be seen that the growth rates of modes with higher $m$ drop more rapidly than growth rates of low- $m$ modes, when $S_{\mathrm{Hp}}$ is increased, which is also reflected by the shift of the peak $\gamma_{\text {peak }}$ to lower $m$. This is important, because the mode number of the fastest-growing mode determines the size of the magnetic islands formed in the early nonlinear regime. ${ }^{4}$

In the remaining part of this section, the $S_{\mathrm{Hp}}$ dependence of DTM growth rates is examined in detail. Comparisons between low- and higher- $m$ modes are made and the role of the distance $D_{12}$ will also be emphasized. First, cases where $q_{\mathrm{s}}=1$ are considered, whereby a distinction is made between DTMs with $m=1$ and $m>1$. The results obtained for $q_{\mathrm{s}}=1$ are readily applied to cases with $q_{\mathrm{s}}>1$, as will be shown at the end of this section where $q_{\mathrm{s}}=2$ DTMs are considered. 


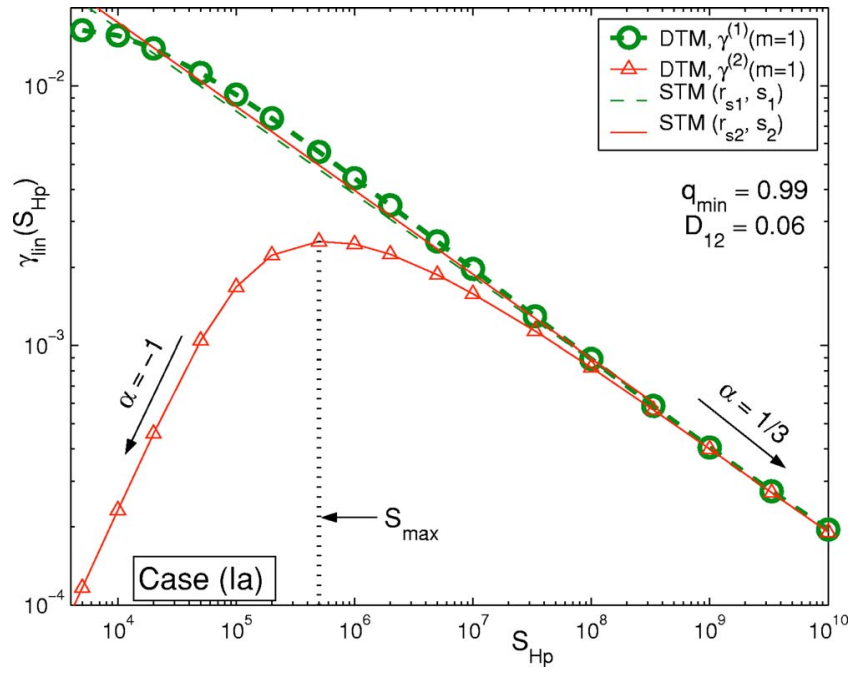

FIG. 11. (Color online). $S_{\mathrm{Hp}}$ dependence of the linear growth rates of the two $m=1$ DTM eigenmodes $M^{(1)}$ and $M^{(2)}$ of Case (Ia) [cf. Fig. 3, (a) and (c)]. For comparison the growth-rate scalings of "corresponding" STMs are shown as well. This means, that the instability of the DTM eigenmode $M^{(1)}$ is compared with the STM mode on the resonant surface $\left(r_{\mathrm{s} 1}, s_{1}\right)$ (monotonic profile with negative shear), while $M^{(2)}$ is compared with the STM mode on $\left(r_{\mathrm{s} 2}, s_{2}\right)$ (positive-shear profile). The STM results are plotted as straight lines, fitted to the actual data. In the regime $S_{\mathrm{Hp}} \gtrsim 10^{6}$, the $\gamma_{\mathrm{lin}} \propto S_{\mathrm{Hp}}^{-\alpha}$ scaling with $\alpha=1 / 3$ is followed closely by both STMs: $\alpha \approx 0.32$.

\section{DTMs with $m=1$}

It is known that DTMs with $m=1$ behave similarly to $m=1$ STMs, regardless of the location of the resonant surfaces and their mutual distance. ${ }^{6}$ Due to this close relationship between the $m=1$ STMs and the $m=1$ DTMs it is possible to decompose the non-monotonic $q$ profile into two monotonic ones-one with a resonance at $r_{\mathrm{s} 1}$ and negative shear $s_{1}$, the other with a resonance at $r_{\mathrm{s} 2}$ and positive shear $s_{2}$ - and compare the growth rates and $S_{\mathrm{Hp}}$ dependences of the STM eigenmodes with the corresponding DTMs. Our aim is to characterize deviations between STMs and DTMs in certain ranges of $S_{\mathrm{Hp}}$. For clarity, both $M^{(1)}$ - and $M^{(2)}$-type eigenmodes in a double-tearing configuration are referred to as "DTMs."

In Fig. 11 this comparison is performed for Case (Ia), where $D_{12}=0.06$ [Fig. 1(a)]. The results for Cases (Ib) and (Ic), where $D_{12}=0.21$ and $D_{13}=0.31$, respectively, are presented in Fig. 12.

In Case (Ia), $\left|s_{1}\right| \approx s_{2}$ (cf. Table II), so that the growth rates of the two STMs are almost equal (Fig. 11). Both have $\alpha=0.32 \approx 1 / 3$, in agreement with linear theory. It can be observed that the DTM growth rates coincide with the corresponding STM growth rates only at relatively high values of $S_{\mathrm{Hp}}: \gamma_{\text {lin }}^{(1)}$ for $S_{\mathrm{Hp}}>10^{7}$ and $\gamma_{\text {lin }}^{(2)}$ for $S_{\mathrm{Hp}}>10^{8}$. The agreement between $m=1$ DTMs and STMs for intermediate $S_{\mathrm{Hp}}$ is significantly improved when the distance between the resonant surfaces is increased. This can be seen by comparing Fig. 11 with Fig. 12.

The linear growth rate of a tearing mode plotted as a function of the magnetic Reynolds number, $\gamma_{\text {lin }}\left(S_{\mathrm{Hp}}\right)$, always has a maximum at a certain value $S_{\mathrm{Hp}}=S_{\mathrm{max}}$. For instance, for $\gamma_{\text {lin }}^{(2)}$ in Fig. 11, one finds $S_{\max } \approx 5 \times 10^{5}$. In the regime where $S_{\mathrm{Hp}}<S_{\max }$ the width of the linear resistive layer $\delta_{\eta}$ [Eq. (22)] is comparable to $D_{12}$. Due to the strong resistive diffusion in the regime $S_{\mathrm{Hp}} \lesssim S_{\max }$, the two resonant surfaces are effectively seen as a single $q_{\mathrm{s}}=1$ "surface." This leads to the reduction in the DTM growth rate apparent in Fig. 11. For $S_{\mathrm{Hp}} \ll S_{\text {max }}$ a linear dependence $\gamma_{\text {lin }} \propto S_{\mathrm{Hp}}$ (i.e., $\left.\alpha=-1\right)$ is obtained independently of $m$.

In summary, for $m=1$ DTMs it is found that for sufficiently small $D_{12}$, the growth rate of the $M^{(2)}$ mode exhibits no scaling law in a wide range of $S_{\mathrm{Hp}}$. For $S_{\mathrm{Hp}} \ll S_{\max }$ a linear dependence is observed and for $S_{\mathrm{Hp}} \gg S_{\max }$ the scaling exponent $\alpha=1 / 3$ is obtained for both eigenmodes. However, the range of $S_{\mathrm{Hp}}$ in which deviations from the $\alpha=1 / 3$ scaling are observed may extend to high $S_{\mathrm{Hp}}$ when $D_{12}$ is small.
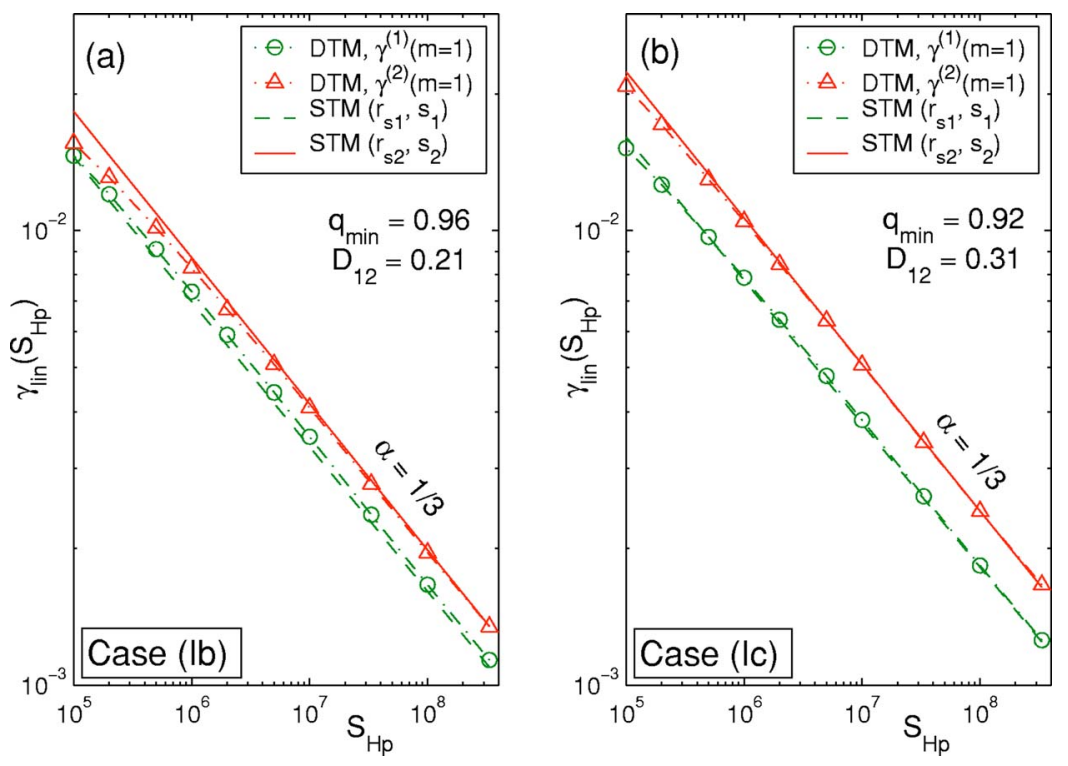

FIG. 12. (Color online). $S_{\mathrm{Hp}}$ dependence of the linear growth rates of the two $m=1$ DTM eigenmodes, (a) for $D_{12}=0.31$ [Case (Ic)] and (b) for $D_{12}=0.21$ [Case (Ib)]. Corresponding STM data are shown as well, as in Fig. 11. 


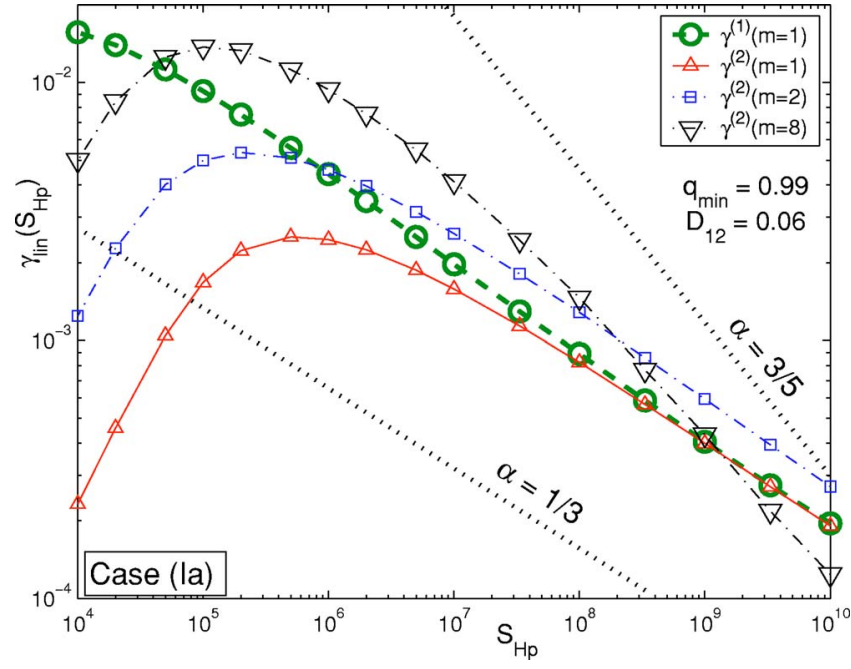

FIG. 13. (Color online). $S_{\mathrm{Hp}}$ dependence of the linear growth rates of $q_{\mathrm{s}}$ $=1$ DTMs with $m=1,2,3$ for a small distance $D_{12}=0.06$ [Case (Ia)]. The dotted lines represent the scaling law $\gamma_{\text {lin }}=S_{\mathrm{Hp}}^{-\alpha}$ with $\alpha=1 / 3$ and $3 / 5$.

\section{DTMs with $m>1$}

Here the $S_{\mathrm{Hp}}$ dependence of $q_{\mathrm{s}}=1$ DTMs with $m>1$ is analyzed. First, consider Case (Ia) where the inter-resonance distance is small: $D_{12}=0.06$. As indicated in Fig. 10, we have selected the growth rates $\gamma_{\operatorname{lin}}^{(2)}(m=2)$ and $\gamma_{\text {lin }}^{(2)}(m=8)$, and plotted them as functions of $S_{\mathrm{Hp}}$ in Fig. 13. It is noted that in Case (Ia) only $M^{(2)}$-type modes are unstable for $m>1$. For comparison, $\gamma_{\text {lin }}^{(1)}(m=1)$ and $\gamma_{\text {lin }}^{(2)}(m=1)$ from Fig. 11 are shown as well. It can be seen that, similarly to the $m=1$ mode, $\gamma_{\text {lin }}^{(2)}(m=2)$ and $\gamma_{\text {lin }}^{(2)}(m=8)$ have $\alpha=-1$ for $S_{\mathrm{Hp}} \ll S_{\max }$, where $S_{\max }(m=2) \approx 10^{5}$ and $S_{\max }(m=8) \approx 2 \times 10^{5}$. In the range of $S_{\mathrm{Hp}}$ shown in Fig. 13, only $\gamma_{\operatorname{lin}}^{(2)}(m=8)$ approaches the scaling $\alpha=3 / 5$ in the limit of high $S_{\mathrm{Hp}}$. The growth rate of the $m=2$ mode, $\gamma_{\operatorname{lin}}^{(2)}(m=2)$, has $\alpha$ close to but somewhat larger than 1/3. Calculations with $m=4$ and $m=6$ (not shown here) gave intermediate values $1 / 3<\alpha_{m}<3 / 5$ (with $\alpha_{m}$ being the scaling exponent for the mode number $m$ in the limit of $S_{\mathrm{Hp}} \gg S_{\max }$ ).

In Fig. 14 the growth rates of the $m=1$ and $m=2$ eigenmodes are compared for Case (Ib), where $D_{12}=0.21$. In contrast to Case (Ia) (Fig. 13, $D_{12}=0.06$ ), now there are two unstable $m=2$ eigenmodes. A scaling exponent $\alpha_{m=2} \approx 3 / 5$ is obtained for both $m=2$ eigenmodes [while $\alpha_{m=2} \approx 1 / 3$ in Case (Ia)].

As a result of the complicated dependence of $\gamma_{\text {lin }}$ on the parameter set $\left\{m, s_{1}, s_{2}, D_{12}, S_{\mathrm{Hp}}\right\}$, growth rates $\gamma_{\text {lin }}(m>1)$ may rise above $\gamma_{\text {lin }}(m=1)$ in certain regimes of the parameter space. This can be seen in Fig. 13: $\gamma_{\text {lin }}^{(2)}(m=8)>\gamma_{\text {lin }}^{(1)}(m=1)$ for $4 \times 10^{4} \leqq S_{\mathrm{Hp}} \leqslant 2 \times 10^{8}$, and $\gamma_{\operatorname{lin}}^{(2)}(m=2)>\gamma_{\operatorname{lin}}^{(1)}(m=1)$ for $S_{\mathrm{Hp}} \gtrsim 8 \times 10^{5}$ (upper limit not known). In this regime dispersion relations are found to peak at $m_{\text {peak }}>1$.

\section{Cases with $q_{\mathrm{s}}=2$}

In Fig. 15 the $S_{\mathrm{Hp}}$ dependence of the linear growth rates of $q_{\mathrm{s}}=2$ DTMs is shown. A case with relatively large $D_{12}$ is plotted in Fig. 15(a). Both $m=2$ eigenmodes follow the scaling law $\gamma_{\text {lin }} \propto S_{\mathrm{Hp}}^{-3 / 5}$ rather well. When $D_{12}$ is reduced the

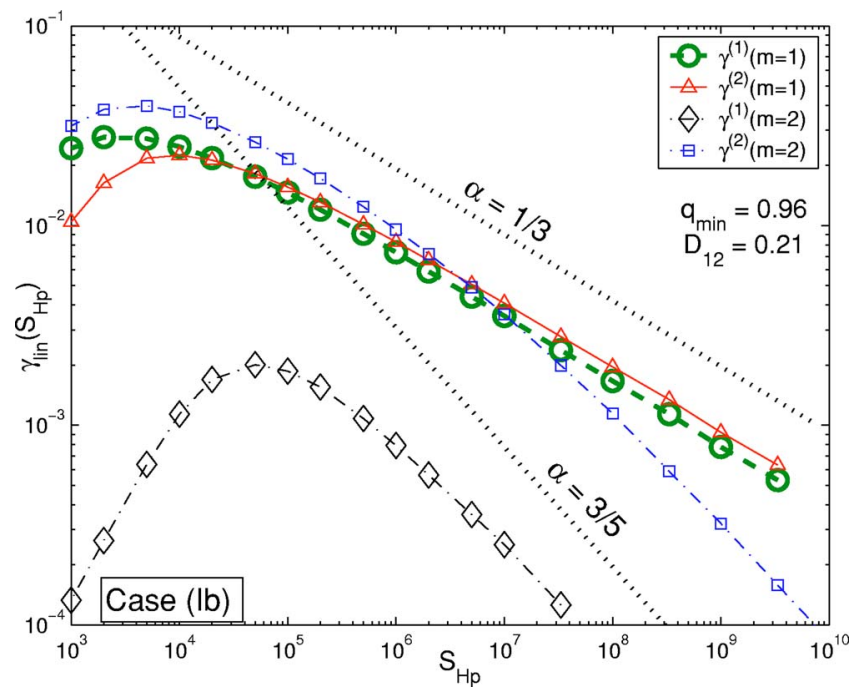

FIG. 14. (Color online). $S_{\mathrm{Hp}}$ dependence of the linear growth rates of $q_{\mathrm{s}}$ $=1$ DTMs with $m=1,2$ for the distance $D_{12}=0.21$ [Case (Ib)].

growth-rate scalings deviate from this power law in a wide range of $S_{\mathrm{Hp}}$, as can be seen in Fig. 15(b). While the higher$m$ mode still approaches $\alpha_{m}=3 / 5$, the lower- $m$ mode has $\alpha_{m}$ close to $1 / 3$. Note the similarity between Fig. 15 and the $q_{\mathrm{s}}=1$ results shown above.

\section{DISCUSSION AND CONCLUSIONS}

Double tearing modes with high poloidal mode numbers are destabilized when the distance between the resonant surfaces is small. For a given inter-resonance distance the mode number of the fastest growing mode was observed to shift to lower $m$ when $S_{\mathrm{Hp}}$ is increased (Fig. 10). This is related to the fact that modes with different mode numbers $m$ approach scaling laws $\gamma_{\mathrm{lin}} \propto S_{\mathrm{Hp}}^{-\alpha}$ with different exponents $\alpha=\alpha_{\mathrm{m}}$. Moreover, and in agreement with earlier works, ${ }^{2,28}$ it was found that the scaling exponent $\alpha_{m}$ is a function of the interresonance distance $D_{12}$ (e.g., Figs. 13 and 14).
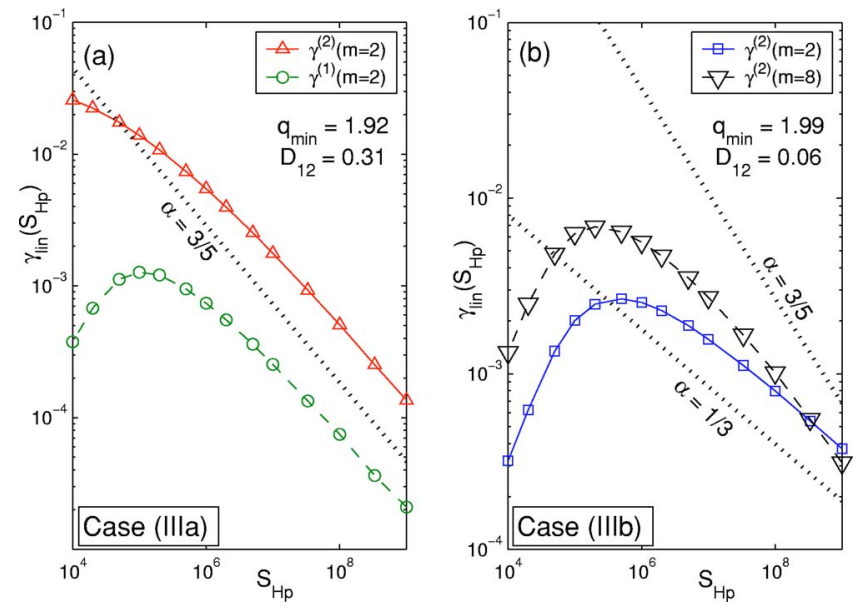

FIG. 15. (Color online). $S_{\mathrm{Hp}}$ dependence of the linear growth rates of $q_{\mathrm{s}}$ $=2$ DTMs. In diagram (a) the growth rates of the two $m=2$ eigenmodes of Case (IIIa) with $D_{12}=0.31$ are shown [cf. Figs. 4(a) and 4(c)]. In (b) the growth rates of the unstable $m=2$ and $m=8$ eigenmodes (both $M^{(2)}$ type) of Case (IIIb) are plotted [cf. Figs. 4(b) and 4(d)]. 
Linear tearing mode theory predicts two characteristic values for the $S_{\mathrm{Hp}}$-scaling exponent: $\alpha=1 / 3$ for $m=1$ modes $^{25}$ and DTMs on nearby resonant surfaces, ${ }^{2}$ and $\alpha$ $=3 / 5$ for STMs. $^{26}$ When the distance $D_{12}$ is increased, DTMs are transformed into STMs and $\alpha_{m}$ gradually increases from $1 / 3$ to $3 / 5$ (except for $m=1$ modes). ${ }^{28}$ In the present work, it was observed that the transition from $\alpha_{m}$ $=1 / 3$ to $3 / 5$ also occurs when $m$ is increased (Figs. 13-15). However, the variation of $m$ does not change the character of the DTM mode structures, which is in contrast with the above-mentioned effect of increasing $D_{12}$ (Figs. 3 and 4).

For practical reasons, the knowledge of the mode number of the dominant mode $m_{\text {peak }}$ in the spectrum $\gamma_{\text {lin }}(m)$ is important, since it determines the structure of the magnetic islands in the early nonlinear regime. ${ }^{4}$ The observations that $m_{\text {peak }}$ varies with $S_{\mathrm{Hp}}$ and that the growth rates $\gamma_{\text {lin }}(m)$ may approach their respective characteristic scaling exponent $\alpha_{m}$ only at very high values of $S_{\mathrm{Hp}}$ [cf. Figs. 13, 14, and 15(b)] have the following important consequence. Results obtained from nonlinear simulations run in the collisional regime (e.g., $S_{\mathrm{Hp}} \sim 10^{6}$ ) for a given configuration may not be easily extrapolated to higher $S_{\mathrm{Hp}}$, since magnetic islands with different poloidal mode numbers are expected for different $S_{\mathrm{Hp}}$. Note, however, that the effective magnetic Reynolds number in the reconnection regions may be smaller due to the action of micro-turbulence (anomalous resistivity). ${ }^{39,40}$

In summary, the linear instability characteristics of DTMs with high poloidal mode numbers $m$ were studied numerically. High- $m$ tearing modes become unstable when two or more resonant surfaces $q\left(r_{\mathrm{s} 1}\right)=q\left(r_{\mathrm{s} 2}\right)=q_{\mathrm{s}}$ are formed in a tokamak plasma and when the distance between these resonances $D_{12}=\left|r_{\mathrm{s} 2}-r_{\mathrm{s} 1}\right|$ is still small. It was shown that despite the low magnetic shear in the vicinity of $q_{\min }$, modes with high $m$ have high growth rates due to the destabilizing effect of small $D_{12}$. The width of the DTM spectrum and mode number of the dominant mode were found to be independent of the $q$ value. Broad spectra of unstable DTMs, with dominant modes having $m>1$, were found in a wide range of magnetic Reynolds numbers, including the regimes in which tokamaks operate. Let us note that this result may also be applied to configurations with more than two resonant surfaces ${ }^{4}$ and configurations with low magnetic shear. ${ }^{41}$

The findings of this linear study motivate a nonlinear investigation of DTMs. Nonlinear simulations were performed in the past for relatively large inter-resonance distances (e.g., Refs. 14, 17, 28, and 38). According to our results, during the stage where the inter-resonance distance is still small, fast growing high- $m$ DTMs may significantly modify the $q$ profile near $q_{\text {min }}$ and thereby affect the longterm evolution. ${ }^{4}$ This might have important implications for the understanding of the sawtooth crash $^{42-44}$ and other applications of DTM dynamics mentioned in the Introduction, such as the anomalous current penetration during the currentramp-up phase, off-axis sawteeth or the formation of ITBs.

An effect that was neglected here but may decouple DTMs in practice is differential rotation. ${ }^{18,31,32}$ However, its influence decreases with decreasing inter-resonance distance. A factor that is expected to be important in regions with low magnetic shear near resonant surfaces (as in the vicinity of $\left.q_{\text {min }}\right)$ is the pressure gradient. ${ }^{45}$ This and other extensions are left for future study.

\section{ACKNOWLEDGMENTS}

A.B. would like to thank Y. Kishimoto for valuable discussions. S.B. acknowledges the Graduate School of Energy Science at Kyoto University for its support and hospitality.

This work is partially supported by the 21 st Century COE Program at Kyoto University.

${ }^{1}$ R. B. White, D. A. Monticello, M. N. Rosenbluth, and B. V. Waddell, in Proceedings of the Conference on Plasma Physics and Controlled Nuclear Fusion Research, Berchtesgaden, Germany, 1976 (International Atomic Energy Agency, Vienna, 1977), Vol. 1, p. 569.

${ }^{2}$ P. L. Pritchett, Y. C. Lee, and J. F. Drake, Phys. Fluids 23, 1368 (1980).

${ }^{3}$ S. M. Mahajan and R. D. Hazeltine, Nucl. Fusion 22, 1191 (1982).

${ }^{4}$ A. Bierwage, S. Hamaguchi, M. Wakatani, S. Benkadda, and X. Leoncini, Phys. Rev. Lett. 94, 065001 (2005).

${ }^{5}$ J. Schmidt and S. Yoshikawa, Phys. Rev. Lett. 26, 753 (1971).

${ }^{6}$ H. P. Furth, P. H. Rutherford, and H. Selberg, Phys. Fluids 16, 1054 (1973).

${ }^{7}$ T. H. Stix, Phys. Rev. Lett. 36, 521 (1976).

${ }^{8}$ D. H. J. Goodall and J. A. Wesson, Plasma Phys. Controlled Nucl. Fusion Res. 26, 789 (1984)

${ }^{9}$ A. W. Edwards, D. J. Campbell, W. W. Engelhardt, H.-U. Fahrbach, R. D. Gill, R. S. Granetz, S. Tsuji, B. J. D. Tubbing, A. Weller, J. Wesson et al., Phys. Rev. Lett. 57, 210 (1986).

${ }^{10}$ G. Taylor, P. C. Efthimion, V. Arunasalam, R. J. Goldston, B. Grek, K. W. Hill, D. W. Johnson, K. McGuire, A. T. Ramsey, and F. J. Stauffer, Nucl. Fusion 26, 339 (1986).

${ }^{11}$ D. J. Campbell, R. D. Gill, C. W. Gowers, J. A. Wesson, D. V. Bartlett, C. H. Best, S. Coda, A. E. Costley, A. Edwards, S. E. Kissel et al., Nucl. Fusion 26, 1085 (1986).

${ }^{12}$ S. B. Kim, Nucl. Fusion 26, 1251 (1986).

${ }^{13}$ S. Ishida, H. Shirai, K. Nakashima, T. Nishitani, T. Fukuda, and JT-60 Team, Plasma Phys. Controlled Fusion 30, 1069 (1988).

${ }^{14}$ B. Carreras, H. R. Hicks, and B. V. Waddell, Nucl. Fusion 19, 583 (1979).

${ }^{15}$ V. V. Parail and G. V. Pereverzev, Sov. J. Plasma Phys. 6, 14 (1980).

${ }^{16}$ W. Pfeiffer, Nucl. Fusion 25, 673 (1985).

${ }^{17}$ Z. Chang, W. Park, E. D. Frederickson, S. H. Batha, M. G. Bell, R. Bell, R. V. Budny, C. E. Bush, A. Janos, F. M. Levinton et al., Phys. Rev. Lett. 77, 3553 (1996).

${ }^{18}$ M. Persson and R. L. Dewar, Phys. Plasmas 1, 1256 (1994).

${ }^{19}$ Y. Ishii, M. Azumi, and Y. Kishimoto, Phys. Rev. Lett. 89, 205002 (2002).

${ }^{20}$ B. V. Waddell, B. Carreras, H. R. Hicks, and J. A. Holmes, Phys. Fluids 22, 896 (1979).

${ }^{21}$ J. W. Connor, S. C. Cowley, R. J. Hastie, T. C. Hender, A. Hood, and T. J. Martin, Phys. Fluids 31, 577 (1988).

${ }^{22}$ S. Günter, S. Schade, M. Maraschek, S. D. Pinches, E. Strumberger, R. Wolf, Q. Yu, and the ASDEX Upgrade Team, Nucl. Fusion 40, 1541 (2000).

${ }^{23}$ I. Voitsekhovitch, X. Garbet, S. Benkadda, P. Beyer, and C. F. Figarella, Phys. Plasmas 9, 4671 (2002).

${ }^{24}$ J. W. Connor, T. Fukuda, X. Garbet, C. Gormezano, V. Mukhavotov, M. Wakatani, the ITB Database Group, and the Topical Group on Transport and Internal Transport Barrier Physics, Nucl. Fusion 44, R1 (2004).

${ }^{25}$ B. Coppi, R. Galvao, R. Pellat, M. N. Rosenbluth, and P. H. Rutherford, Fiz. Plazmy 2, 961 (1976) [Sov. J. Plasma Phys. 2, 533 (1976)].

${ }^{26}$ H. P. Furth, J. Killeen, and M. N. Rosenbluth, Phys. Fluids 6, 459 (1963).

${ }^{27}$ Q. Yu, Phys. Plasmas 3, 2898 (1996).

${ }^{28}$ Y. Ishii, M. Azumi, G. Kurita, and T. Tuda, Phys. Plasmas 7, 4477 (2000).

${ }^{29}$ J. Q. Dong, S. M. Mahajan, and W. Horton, Phys. Plasmas 10, 3151 (2003).

${ }^{30}$ E. D. Held, J. N. Leboeuf, and B. A. Carreras, Phys. Plasmas 6, 837 (1999).

${ }^{31}$ L. Ofman, Phys. Fluids B 4, 2751 (1992).

${ }^{32}$ C. Shen and Z. X. Liu, Plasma Phys. Controlled Fusion 40, 1 (1998).

${ }^{33}$ S. Günter, G. Giruzzi, A. Gude, R. J. L. Haye, K. Lackner, M. Maraschek, S. Schade, S. Sesnic, R. Wolf, Q. Yu et al., Plasma Phys. Controlled Fusion 41, B231 (1999).

${ }^{34}$ R. D. Hazeltine, H. R. Strauss, S. M. Mahajan, and D. W. Ross, Phys. 
Fluids 22, 1932 (1979).

${ }^{35}$ H. R. Strauss, Phys. Fluids 19, 134 (1976).

${ }^{36}$ K. Nishikawa and M. Wakatani, Plasma Physics (Springer, Berlin, 2000).

${ }^{37}$ W. Kerner and H. Tasso, Plasma Phys. 24, 97 (1982).

${ }^{38}$ M. Sato, S. Hamaguchi, and M. Wakatani, J. Phys. Soc. Jpn. 70, 2578 (2001).

${ }^{39}$ H. Ji, M. Yamada, S. Hsu, and R. Kulsrud, Phys. Rev. Lett. 80, 3256 (1998).
${ }^{40}$ J. A. Breslau and S. C. Jardin, Plasma Phys. 10, 1291 (2003).

${ }^{41}$ R. G. Kleva, J. F. Drake, and R. E. Denton, Phys. Fluids 30, 2119 (1987).

${ }^{42}$ F. Porcelli, D. Boucher, and M. N. Rosenbluth, Plasma Phys. Controlled Fusion 38, 2163 (1996).

${ }^{43}$ R. J. Hastie, Astrophys. Space Sci. 256, 177 (1998).

${ }^{44}$ S.-I. Itoh, K. Itoh, H. Zushi, and A. Fukuyama, Plasma Phys. Controlled Fusion 40, 879 (1998).

${ }^{45}$ F. L. Waelbroeck and R. D. Hazeltine, Phys. Fluids 31, 1217 (1988). 